\title{
BIOATIVIDADE DE MACERADOS DE ANTHEMIS SP., CORIANDRUM SATIVUM E PIPER NIGRUM CONTRA SITOPHILUS ZEAMAIS (COLEOPTERA: CURCULIONIDAE)
}

\author{
Scopel W, ${ }^{*}$ Scopel EL, ${ }^{\dagger}$ Botteon VW, ${ }^{\ddagger}$ Roza-Gomes MF§
}

\begin{abstract}
Resumo
Plantas podem apresentar propriedades bioativas inseticidas como mecanismo de defesa natural contra algumas espécies de insetos-praga. Visando investigar possíveis fontes naturais que contribuam para a proteção de grãos armazenados, foram avaliadas as propriedades de repelência e inseticida de macerados de Anthemis sp., Coriandrum sativum e Piper nigrum sobre o gorgulho-do-milho, Sitophilus zeamais Motschulsky, 1855 (Coleoptera: Curculionidae). Dois experimentos foram instalados com delineamento inteiramente casualizado, utilizando-se dois cultivares de milho híbrido (AS 1551 YG e AS 1555 YG) como substratos alimentares. O primeiro experimento objetivou verificar a mortalidade da praga após sete dias de exposição aos vegetais estudados, com delineamento em arranjo fatorial (testemunha; três espécies vegetais; dois cultivares de milho). No segundo

\footnotetext{
* Doutora em Ciências (Entomologia) pela Universidade de São Paulo; Mestre em Agronomia (Produção Vegetal) pela Universidade de Passo Fundo; Avenida Pádua Dias, 11, Agronomia, 13418900, Piracicaba, São Paulo, Brasil; wanessa_scopel@yahoo.com.br

† Graduado em Agronomia e Pós-graduado em Engenharia de Segurança do Trabalho pela Universidade do Oeste de Santa Catarina; scopel_smo@hotmail.com

¥ Mestre em Ciências pelo Centro de Energia Nuclear na Agricultura da Universidade de São Paulo; Graduado em Ciências Biológicas pela Universidade de São Paulo; victor_botteon2@hotmail.com $\S$ Mestre e Doutora em Agronomia (Produção Vegetal) pela Universidade de Passo Fundo; Graduada em Ciência Biológicas e Agronomia pela Universidade do Oeste de Santa Catarina; Professora na Universidade do Oeste de Santa Catarina; margarida.gomes@unoesc.edu.br
} 
experimento, avaliou-se a repelência da praga promovida pelas espécies vegetais, testadas isoladamente em uma arena. Após 24 horas, a porcentagem de adultos repelidos (\% AR) foi calculada. $O$ estudo permitiu verificar que $P$. nigrum apresenta propriedade inseticida, observando uma mortalidade média de 49\%. Anthemis sp. demonstrou efeito repelente (I.R./I.D. $=0,44$ ) sobre S. zeamais quando o substrato utilizado foi o híbrido AS 1555 YG, com 26,5\% de adultos repelidos. Já para o híbrido AS 1551 YG, P. nigrum mostrou efeito repelente (I.R./I.D. = 0,68 e 31\% de adultos repelidos), enquanto $C$. sativum e Anthemis sp. foram consideradas neutras. Palavras-chave: Pragas de grãos armazenados. Manejo integrado de pragas. Plantas inseticidas.

\title{
Bioactivity of macerates of Anthemis sp., Coriandrum sativum and Piper nigrum against Sitophilus zeamais (Coleoptera: Curculionidae)
}

\begin{abstract}
Plants may exhibit insecticide bioactive properties as a natural defense mechanism against some insect-pest species. The insect repellency and insecticidal properties of Anthemis sp., Coriandrum sativum and Piper nigrum against the maize weevil, Sitophilus zeamais Motschulsky, 1855 (Coleoptera: Curculionidae), were investigated in order to find possible natural sources to allow the protection of stored grains. Two experiments were installed with a completely randomized design, using two cultivars of hybrid maize (AS 1551 YG and AS 1555 YG) as food substrates. The first experiment aimed to verify the mortality of the pest after 7 days of exposure to the studied plants at $0.5 \mathrm{~g} /$ treatment, with a factorial arrangement (control, three plant species, two maize cultivars). In the second experiment, the pest repellency promoted by each plant species was evaluated in an arena. After 24 hours, the percentage of adults repelled (\% AR) was calculated. The study showed that $P$. nigrum presented insecticidal properties, observing a mean mortality of 49\%. Anthemis sp. presented a repellent effect (I.R./I.D. =0.44) on S. zeamais when the substrate used was the hybrid AS 1555 YG, with 26.5\% adults repelled. As for the hybrid AS 1551 YG, P. nigrum showed a repellent effect (I.R./I.D. $=0.68$ and $31 \%$ of adults repelled), while C. sativum and Anthemis sp. were considered neutral against the maize weevil.
\end{abstract}

Keywords: Stored grain pests. Integrated pest management. Insecticidal plants.

\section{INTRODUÇÃO}

O milho (Zea mays L.) é um importante produto agrícola que sofre com perdas provocadas por diversos fatores, antes, durante e após a colheita. ${ }^{1,2}$ As perdas quantitativas anuais causadas por pragas durante o período de armazenamento de grãos são da ordem de $10 \%$ da produção mundial, valor referido apenas aos 
danos diretos causados pelos insetos ao embrião e ao endosperma das sementes; desconsideram-se as perdas qualitativas oriundas de danos indiretos, como injúrias causadas por agentes patogênicos, o aquecimento da massa de grãos decorrente da atividade dos insetos e a consequente redução do valor nutritivo. ${ }^{3-6}$

Os gorgulhos (Coleoptera) são insetos de grande importância agrícola, pois podem danificar os grãos no período pós-colheita, comprometendo a comercialização e a viabilidade econômica do produto. Entre as principais pragas de grãos de milho, durante o período de armazenamento, destacam-se Sitophilus zeamais (Mots.), S. oryzae (L.) (Coleoptera: Curculionidae) e Rhyzopertha dominica (Fab.) (Coleoptera: Bostrichidae), sendo consideradas pragas primárias por terem a capacidade de perfurar o tegumento de grãos e sementes. ${ }^{7} \mathrm{O}$ gorgulho-domilho, S. zeamais, é considerado uma das principais pragas do milho estocado, frequentemente encontrado em outros grãos de importância econômica. ${ }^{8}$ A postura é realizada no interior dos grãos, onde as larvas e pupas se desenvolvem. Já a infestação inicia-se no campo e continua nas unidades de armazenamento, situação em que o processo de secagem deixa os grãos suscetíveis à infestação e dificulta a aplicação de produtos inseticidas. ${ }^{9-12}$

O principal método de controle empregado contra essa praga é o controle químico efetuado com inseticidas sintéticos e fumegantes, os quais podem causar intoxicações aos aplicadores, apresentar persistência de resíduos tóxicos nos grãos e resultar na seleção de populações de insetos resistentes. ${ }^{13}$ Dessa forma, métodos alternativos de controle têm sido testados no contexto de manejo integrado de pragas (MIP), buscando a utilização de compostos menos tóxicos, acessíveis e de menor custo, como a utilização de pós, extratos aquosos ou orgânicos e óleos essenciais ou emulsionáveis derivados de fontes naturais. ${ }^{14-16}$ Esses compostos apresentam toxicidade por contato, ingestão e/ou fumigação, podendo afetar o desenvolvimento dos insetos e provocar repelência, deterrência na alimentação e oviposição, e até mesmo mortalidade, não promovendo a seleção de insetos resistentes. ${ }^{14,17,18}$

Atualmente, o uso de diversos inseticidas não é mais permitido em vários países, por apresentarem altos níveis de toxicidade ou por serem persistentes no ambiente. ${ }^{19,20}$ Essa proibição vem motivando a busca por novas moléculas, não somente na promoção da agricultura orgânica e sustentável, ${ }^{15,16}$ mas também em amplas áreas de cultivo em razão da diminuição da disponibilidade de moléculas sintéticas com ação inseticida presentes no mercado. ${ }^{19,21} \mathrm{Na}$ interação insetoplanta, as plantas produzem metabólitos secundários que, em decorrência da toxicidade de determinados compostos, podem atuar como meios de defesas naturais contra a herbivoria. ${ }^{15,22}$ Esses aleloquímicos bioinseticidas compreendem uma gama diversificada de compostos que podem constituir uma importante fonte de moléculas a serem utilizadas em programas de MIP em grãos armazenados. ${ }^{22-24}$ 
A utilização de plantas inseticidas no controle de gorgulhos vem sendo estudada nos últimos anos. Oliveira e Vendramim ${ }^{25}$ avaliaram a repelência de adultos de Zabrotes subfasciatus em sementes de feijoeiro utilizando pó de Piper nigrum (pimenta-do-reino) nas concentrações de 2,5 e 5,0\% e verificaramrepelência, respectivamente, de 35,9 e 45,4\%, concluindo que a pimenta-do-reino moída constitui uma fonte promissora de inseticida natural. Em outro estudo, Mazzonetto e Vendramim ${ }^{26}$ constataram que o pó das folhas de Coriandrum sativum (coentro) não foi repelente sobre Acanthoscelides obtectus em feijão armazenado, mas provocou a mortalidade total dos indivíduos adultos. Já em um estudo visando ao controle de $S$. zeamais, outros autores ${ }^{24}$ identificaram importantes compostos bioinseticidas derivados de Annona mucosa Jacq. (Annonaceae), conhecida comumente como araticum ou fruta-do-conde.

Esses resultados, dentre outros, estimularam a realização de pesquisas sobre novos aleloquímicos e atualmente aparecem como uma ferramenta muito promissora no controle de pragas, podendo facilmente ser incluídos no MIP. Nesse contexto, novas fontes promissoras de compostos inseticidas de origem vegetal precisam ser investigadas para serem utilizadas como agentes protetores de grãos armazenados. Desse modo, neste estudo objetivou-se avaliar a existência ou não de efeito inseticida e/ou de repelência de espécies vegetais ainda pouco exploradas, Anthemis sp. (Asteraceae), Coriandrum sativum (Apiaceae) e Piper nigrum (Piperaceae), contra S. zeamais em dois híbridos de milho (AS 1551 YG e AS 1555 YG).

\section{MATERIAL E MÉTODOS}

Os experimentos foram instalados e conduzidos no Laboratório de Zoologia da Universidade do Oeste de Santa Catarina (Unoesc) de São Miguel do Oeste, SC, à temperatura de $25 \pm 2^{\circ} \mathrm{C}$, umidade relativa de $60 \pm 10 \%$ e fotoperíodo de 14:10 horas.

\subsection{ESTABELECIMENTO E MANUTENÇÃO DA CRIAÇÃO DE SITOPHILUS ZEAMAIS}

A criação de $S$. zeamais foi estabelecida a partir de exemplares obtidos de uma população mantida no Laboratório de Entomologia da Embrapa Trigo, em Passo Fundo, RS.

Os insetos utilizados nos ensaios foram criados em frascos de vidro com capacidade para $500 \mathrm{~cm}^{3}$, com tampa perfurada e revestida internamente com tecido fino (voile). Grãos de milho, com teor de umidade de $13 \%$ e expostos, previamente, $\mathrm{a}-10^{\circ} \mathrm{C}$ durante 48 horas, para eliminar insetos contaminantes, foram utilizados como substrato para a manutenção da criação de $S$. zeamais. Os insetos foram confinados nos recipientes durante 10 dias para efetuarem a postura e, em seguida, retirados. Os recipientes foram armazenados em sala climatizada até a emergência de novos adultos, os quais foram selecionados com o auxílio de peneira e pincel para serem utilizados nos ensaios. 


\subsection{MATERIAL VEGETAL}

Dois cultivares de milho foram utilizados como substrato alimentar dos gorgulhos-do-milho: híbrido 1 (AS 1551 YG), caracterizado como grãos semiduros e amarelados, e híbrido 2 (AS 1555 YG), caracterizado como grãos duros e alaranjados.

Três espécies botânicas foram testadas quanto à bioatividade contra S. zeamais: Anthemis sp. (macela) (Asteraceae), Coriandrum sativum (coentro) (Apiaceae) e Piper nigrum (pimenta-do-reino) (Piperaceae). As plantas foram adquiridas na Distribuidora de Produtos Brusto Ltda., localizada em São Miguel do Oeste, SC.

material vegetal passou por um processo de triagem para remoção das impurezas. Em seguida, foi macerado em almofariz com auxílio de pistilo, peneirado (malha $2 \mathrm{~mm}$ ) para padronização da granulometria, sendo, em seguida, verificada a massa de cada material em balança analítica de precisão 0,01 g. Devido às referências de potenciais propriedades inseticidas, a estrutura de Anthemis sp. submetida à maceração foi a flor, e no caso de C. sativum e P. nigrum, foram maceradas as sementes. ${ }^{26-29}$

\subsection{EFEITO DOS VEGETAIS SOBRE A MORTALIDADE DE ADULTOS DE SITOPHILUS ZEAMAIS}

Trinta gramas de grãos de milho limpos e secos foram inseridos no interior de recipientes plásticos com capacidade para $250 \mathrm{~cm}^{3}$ e misturados com 0,5 g/ tratamento dos macerados vegetais. Os recipientes foram fechados com tampas plásticas e agitados manualmente durante dois minutos para homogeneizar a mistura.

Cada parcela foi infestada com 20 adultos não sexados de S. zeamais em um delineamento experimental inteiramente casualizado, com arranjo dos tratamentos em esquema fatorial 4x2 (tratamentos $x$ híbridos de milho) com cinco repetições. Decorridos sete dias após o acondicionamento, efetuou-se a abertura dos recipientes para a contagem dos insetos mortos. Foram considerados vivos todos os insetos que moviam qualquer estrutura do corpo, mesmo aqueles que apresentaram comportamento de imobilidade quando perturbados. Os valores de mortalidade de adultos de S. zeamais foram expressos em porcentagem (\%).

Os resultados experimentais foram submetidos à análise de variância (ANOVA), e as médias comparadas pelo teste de Tukey ao nível de 5\% de probabilidade, com auxílio do programa estatístico SISVAR. ${ }^{30}$

\subsection{EFEITOS DAS PLANTAS SOBRE A REPELÊNCIA DE ADULTOS DE SITOPHILUS ZEAMAIS}

A repelência das espécies vegetais sobre os adultos de $S$. zeamais foi avaliada em um experimento com delineamento inteiramente casualizado. Cada espécie vegetal (Anthemis sp., C. sativum e P. nigrum) foi testada separadamente, utilizando-se uma arena elaborada com três recipientes plásticos cilíndricos de dimensões de $7 \mathrm{~cm}$ de diâmetro e $5 \mathrm{~cm}$ de altura, sendo um central interligado 
aos demais por meio de cilindros plásticos de 1,5 cm de diâmetro (Figura 1). No recipiente testemunha (A) foi inserido apenas o substrato alimentar (milho); no recipiente $C$ foram adicionados $30 \mathrm{~g}$ de milho misturados com 0,5 g do macerado da espécie vegetal em teste; no recipiente central B foram liberados 20 adultos não sexados. Após 24 horas da liberação dos besouros, foi contado o número de insetos presente na testemunha, representando a porcentagem de adultos repelidos por cada espécie botânica (\% AR).

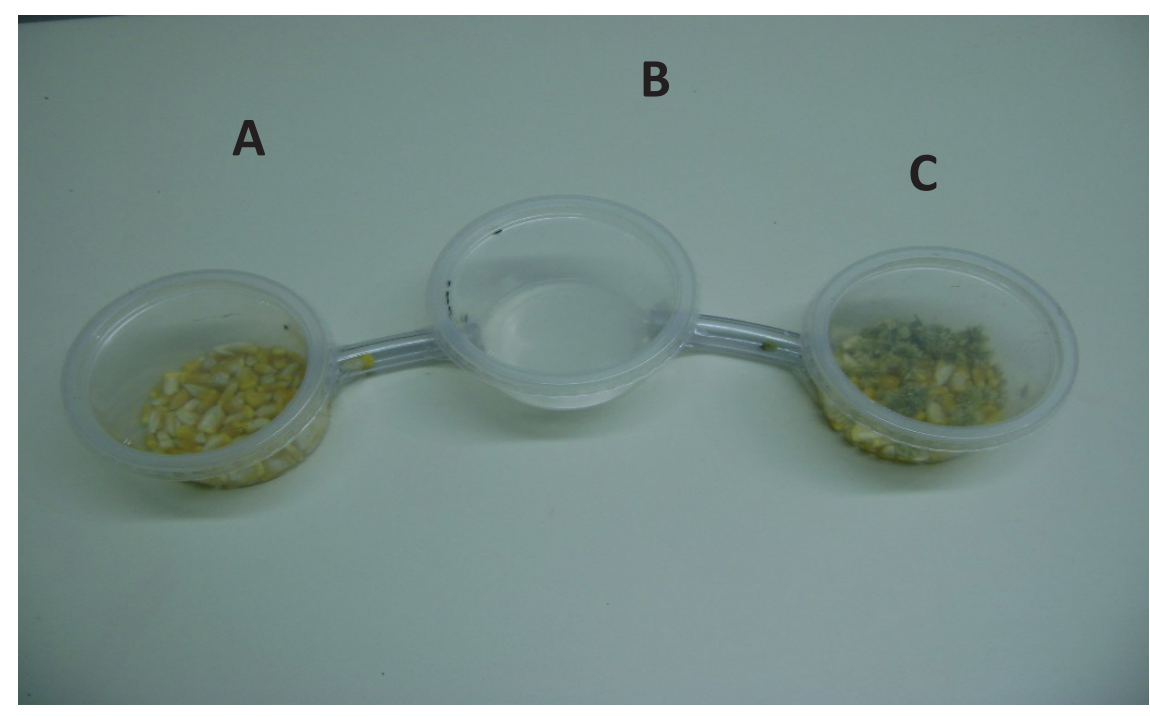

Figura 1 - Arena utilizada nos testes de repelência.ll

Para comparação dos diversos tratamentos, foi estabelecido um índice de repelência/deterrência (I.R./I.D.) adaptado de Lin, Kogan e Fisher, ${ }^{31}$ calculado pela fórmula I.R./I.D.= 2G/(G+P), em que $G=\%$ de insetos nos grãos tratados com o macerado em teste e $P=\%$ de insetos nos grãos controle (testemunha). Com base no I.R./I.D. e no desvio padrão obtidos, determinou-se o intervalo de classificação (l. Class.) para as médias dos tratamentos pela fórmula: I. Class. $1 \pm t\left({ }_{n-1 ; a: 0,05}\right) \times(D P / \sqrt{ } n)$; em que $t=$ valor de " $t$ " tabelado; $D P=$ desvio padrão; $n$ = número de repetições.

Os macerados foram considerados neutros quando o valor de I.R./I.D. esteve compreendido dentro do I. Class. avaliado; repelente e/ou deterrente quando o valor do I.R./I.D. foi inferior ao menor valor obtido para o I. Class.; e atraente e/ou fagodeterrente quando o I.R./I.D. foi superior ao maior I. Class. calculado.

Assim como no teste de mortalidade, os resultados de adultos repelidos foram analisados estatisticamente pelo teste $F$, sendo as médias comparadas pelo teste de Tukey a 5\% de probabilidade, utilizando o programa estatístico SISVAR. ${ }^{30}$

\footnotetext{
\| A: testemunha (milho). B: local de liberação de 20 adultos não sexados de Sitophilus zeamais. C: milho misturados com 0,5 $\mathrm{g}$ do macerado de uma das espécies vegetais.
} 


\section{RESULTADOS E DISCUSSÃO}

Avaliando-se o efeito inseticida dos macerados obtidos das espécies vegetais avaliadas, não foi constatada diferença estatística para o fator híbrido de milho nem para a interação entre híbrido e espécies botânicas (Tabela 1).

Tabela 1 - Mortalidade (\%) de S. zeamais após sete dias confinados com espécies vegetais em dois híbridos de milho"

\begin{tabular}{lrrr}
\hline Espécie & $\begin{array}{r}\text { Híbrido 1 (AS } \\
\mathbf{1 5 5 1} \text { YG) }\end{array}$ & $\begin{array}{r}\text { Híbrido 2 (AS } \\
\mathbf{1 5 5 5} \text { YG) }\end{array}$ & Média \\
\hline \multicolumn{4}{c}{---- Mortalidade (\%) } \\
\hline C. sativum & ---- \\
\hline Anthemis sp. & 2 & 3 & $2,5 \mathrm{b*}$ \\
P. nigrum & 0 & 0 & $0 \mathrm{~b}$ \\
Testemunha & 45 & 53 & $49 \mathrm{a}$ \\
\hline Média & 0 & 0 & $0 \mathrm{~b}$ \\
\hline CV (\%) & $11,7 \mathrm{~A}$ & $14 \mathrm{~A}$ \\
\hline
\end{tabular}

Neste estudo, não foi verificado efeito inseticida de Anthemis sp. e C. sativum sobre $S$. zeamais em razão dos baixos valores de mortalidade observados, sendo 0 e 2,5\%, respectivamente. O híbrido 2 (AS 1555 YG), embora não tenha diferido estatisticamente do híbrido 1 (AS 1551 YG), apresentou maior mortalidade dos insetos em estudo (Tabela 1). Isso pode ter ocorrido em decorrência do tegumento duro característico desse cultivar e, possivelmente, pela dificuldade de perfurar o grão para se alojar em seu interior, ficando expostos por um maior período de tempo à ação inseticida. A aplicação de $3 \mathrm{~mL}$ dos extratos de P. nigrum, Anthemis sp. e C. sativum diretamente sobre o noto de Sitophilus spp. após 48 horas, causou $20 \%, 10 \%$ e $0 \%$ de mortalidade, respectivamente, e quando aplicados por meio de vaporização, a mortalidade foi maior: $100 \%, 96,75 \%$ e $95,87 \%$, respectivamente, observando variação na mortalidade da praga dependendo do método de aplicação.

Já para o macerado de P. nigrum, observou-se uma média de mortalidade de $49 \%$, evidenciando efeito inseticida. Diversos estudos demonstraram a eficácia de extratos de plantas do gênero Piper para o controle de pragas de grãos armazenados, principalmente em razão da presença de substâncias como as piperamidas. ${ }^{32} \mathrm{~A}$ taxa de mortalidade de S. zeamais foi diretamente proporcional à concentração de extrato de semente de $P$. nigrum e o tempo de exposição, alcançando $100 \%$ de mortalidade em 135 min de exposição a $0,298 \mathrm{mg} / \mathrm{mL}$ do extrato..$^{33}$

No teste de repelência, baseando-se no índice de repelência e/ou deterrência, o macerado de P. nigrum (I.R./I.D. I.P. inferior ao menor valor obtido para o I. Class.)

\footnotetext{
"Médias seguidas pelas mesmas letras maiúsculas na horizontal e letras minúsculas na vertical não diferem pelo teste de Tukey a $5 \%$ de probabilidade.
} 
foi o macerado que mais repeliu os adultos de S. zeamais em contato com o híbrido de milho AS 1551 YG. Já Anthemis sp. e C. sativum apresentaram efeito considerado neutro (Tabela 2).

Tabela 2 - Porcentagem de adultos repelidos (\% AR) e índice de repelência e/ou deterrência (I.R./ I.D.) de S. zeamais em arenas contendo grãos de milho do híbrido AS 1551 YG tratados e não tratados com macerados vegetais**

\begin{tabular}{lrrrr}
\hline Espécie & \% AR & $\begin{array}{r}\text { I.R./I.D. }{ }^{\text {1a }} \\
\text { (Média } \pm D P)\end{array}$ & I. Class. $^{2 b}$ & Classificação \\
\hline C. sativum & $33,5^{\text {ns }}$ & $0,9 \pm 0,41$ & $1 \pm 0,33$ & Neutro \\
\hline $\begin{array}{l}\text { Anthemis } \\
\text { sp. }\end{array}$ & $28,5^{\text {ns }}$ & $0,85 \pm 0,45$ & $1 \pm 0,36$ & Neutro \\
\cline { 2 - 5 } $\begin{array}{l}\text { P. nigrum } \\
\text { Média }\end{array}$ & $31^{\text {ns }}$ & $0,68 \pm 0,26$ & $1 \pm 0,21$ & Repelente \\
\cline { 2 - 5 } CV (\%) & 31 & & & \\
\hline
\end{tabular}

Não foi constatada diferença significativa das espécies em estudo para a variável porcentagem de adultos repelidos (\% AR), sendo que esta variou de $28,5 \%$ (Anthemis sp.) a 33,5\% (C. sativum).

No teste em que foi utilizado como substrato de alimentação o híbrido de milho AS 1555 YG, verificou-se o efeito repelente somente do macerado de Anthemis sp. sobre os adultos de S. zeamais (Tabela 3).

Tabela 3 - Porcentagem de adultos repelidos (\% AR) e índice de repelência e/ou deterrência (I.R./ I.D.) de S. zeamais em arenas contendo grãos de milho do híbrido AS 1555 YG tratados e não tratados com macerados vegetaistł

\begin{tabular}{|c|c|c|c|c|}
\hline Espécie & $\% A R$ & $\begin{array}{r}\text { I.R./I.D.a } \\
\text { (Média土DP) }\end{array}$ & I. Class. ${ }^{b}$ & Classificação \\
\hline C. sativum & $\begin{array}{r}18,0 \\
b\end{array}$ & $0,77 \pm 0,32$ & $1 \pm 0,25$ & Neutro \\
\hline Anthemis sp. & $\begin{array}{r}26,5 \\
a b \\
\end{array}$ & $0,44 \pm 0,38$ & $1 \pm 0,30$ & Repelente \\
\hline P. nigrum & $\begin{array}{r}34,5 \\
a\end{array}$ & $1,19 \pm 0,42$ & $1 \pm 0,34$ & Neutro \\
\hline Média & 47,84 & & & \\
\hline CV (\%) & 49,45 & & & \\
\hline
\end{tabular}

A espécie $P$. nigrum apresentou a maior porcentagem de adultos de $S$. zeamais repelidos em arenas com híbrido AS 1555 YG, ficando 8,2\% acima da média, porém não diferiu estatisticamente de Anthemis sp. (26,5\% de AR). Já C. sativum foi considerada uma espécie neutra,34 sendo comprovado pelo fato de apresentar a menor porcentagem de adultos repelidos (18\%).

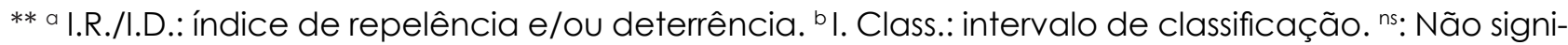
ficativo ao nível de $5 \%$ de probabilidade.

t† al.R./I.D.: índice de repelência e/ou deterrência. ${ }^{b}$ I. Class.: intervalo de classificação.
} 
Observou-se, também, efeito de repelência sobre S. zeamais utilizando extrato de $P$. nigrum com concentração entre 0,5 e $2 \%$, com índice de repelência variando entre 0,75 e 0,77.35 Mishra e Tripathi36 verificaram uma repelência de até $83,3 \%$ utilizando óleos essenciais derivados de C. sativum a $0,2 \%$ de concentração contra S. oryzae. Dados quanto à porcentagem de adultos repelidos e ao índice de repelência e/ou deterrência de Anthemis sp. sobre insetos ainda são escassos na literatura, restringindo-se apenas à variável mortalidade.

Os inseticidas botânicos contêm inúmeros compostos biologicamente ativos capazes de interações diversas sobre populações de pragas, e precisam ser investigados para auxiliar na formulação de novos e eficientes produtos.22,37 O emprego dessas plantas, principalmente na forma de pó seco, favorece especialmente o pequeno produtor por proporcionar menor custo, facilidade de obtenção e aplicação e não exigindo pessoal com alto grau de qualificação técnica. Além disso, as plantas podem ser cultivadas na propriedade, facilitando a sua utilização. 26

Estudos como este são importantes no contexto de programas de MIP, uma vez que a identificação de novos aleloquímicos pode levar ao desenvolvimento de produtos mais eficazes para a proteção de produtos armazenados.38 Outros resultados39 demostraram que a bioatividade de derivados vegetais contra $S$. zeamais pode variar em virtude de diversos fatores, como o método de extração empregado, o tipo de produto e a concentração utilizada, e também pelo tempo de contaminação em que o inseto-alvo permanece exposto. Em razão disso, estudos futuros devem ser realizados para se avaliar o potencial inseticida dessas espécies vegetais sobre S. zeamais com diferentes métodos de extração, diferentes estruturas das plantas, a determinação dos grupos funcionais desses compostos, o efeito sobre inimigos naturais, bem como a possível aplicabilidade prática no campo.

\section{CONCLUSÃO}

Considerando as espécies vegetais testadas, o método de aplicação, o tempo de exposição e a concentração utilizada, P. nigrum apresentou propriedade inseticida sobre adultos de $S$. zeamais.

Anthemis sp. apresentou efeito repelente sobre S. zeamais quando o substrato utilizado foi o híbrido AS 1555 YG. Já para o híbrido AS 1551 YG, apenas P. nigrum mostrou efeito repelente, enquanto C. sativum e Anthemis sp. foram consideradas neutras.

\section{Agradecimentos}

A Albeneir Didomênico, representante da empresa Agroeste Sementes, pelo fornecimento dos cultivares de milho. 


\section{REFERÊNCIAS}

1. Tavares MAGC. Bioatividade da erva-de-santa-maria, Chenopodium ambrosioides L. (Chenopodiaceae), em relação a Sitophilus zeamais Mots., 1855 (Col.: Curculionidae) [dissertação]. Piracicaba: Escola Superior de Agricultura "Luiz de Queiroz", Universidade de São Paulo; 2002.

2. Coitinho RLBC. Atividade inseticida de óleos essenciais sobre Sitophilus zeamais Mots (Coleoptera: Curculionidae) [tese]. Recife: Universidade Federal Rural de Pernambuco; 2009.

3. Lorini I. Controle integrado de pragas de grãos armazenados. Passo Fundo: EMBRAPA-CNPT; 1998.

4. Moreira MAB, Zarbin PHG, Coracini MDA. Feromônios associados aos coleópteros-praga de produtos armazenados. Quím Nova. 2005; 28(3):472-7.

5. Neethirajan S, Karunakaran C, Jayas DS, White NDG. Detection techniques for stored-product insects in grain. Food Control. 2007; 18(2):157-62. doi:10.1016/j. foodcont.2005.09.008

6. Lazzari SMN, Lazzari FA. Insetos-praga de grãos armazenados. In Panizzi AR, Parra JRP, editores. Bioecologia e nutrição de insetos. Brasília: Embrapa Informação Tecnológica; 2009. p. 667-732.

7. Pereira PRVS, Furiatti RS, Lazzari FA, Pinto-Júnior AR. Avaliação de inseticidas no controle de Sitophilus oryzae (L.) (Coleoptera: Curculionidae), e Rhyzopertha dominica (Fab.) (Coleoptera: Bostrichidae) em milho armazenado. An. Soc. Entomol. Bras. 1997; 26(3):41 1-6. doi:10.1590/S0301-80591997000300001

8. Kehinde K, Angela OJ. Comparative biological activity of Synzygium aromaticum (L.) and Xylopia ethiopica on Rhizopertha dominica F. (Coleoptera: Bostrychidae) and Sitophilus zeamais Motsch. (Coleoptera: Cuculionidae) 
in maize grains. J Asia Pac Entomol. 2004; 7(3):339-42. doi: 10.1016/S12268615(08)60236-8

9. Dari S, Pixley KV, Setimela P. Resistance of early generation maize inbred lines and their hybrids to maize weevil [Sitophilus zeamais (Motschulsky)]. Crop Sci. 2010; 50(4):1310-7. doi:10.2135/cropsci2009.10.0621

10.Huang YZ, Hua HX, Li SG, Yang CJ. Contact and fumigant toxicities of calamusenone isolated from Acorus gramineus rhizome against adults of Sitophilus zeamais and Rhizopertha dominica. Insect Sci. 201 1; 18(2):181-8. doi:10.1111/j.1744-7917.2010.01358.x

11.Tefera T, Mugo S, Likhayo P. Effects of insect population density and storage time on grain damage and weight loss in maize due to the maize weevil Sitophilus zeamais and the larger grain borer Prostephanus truncatus. Afr. J. Agric. Res. 2011; 6(10):2249-54. doi:10.5897/AJAR1 1.179

12.Fouad HA, Faroni LRD, Tavares WD, Ribeiro RC, Freitas SD, Zanuncio JC. Botanical extracts of plants from the Brazilian Cerrado for the integrated management of Sitotroga cerealella (Lepidoptera: Gelechiidae) in stored grain. J Stored Prod Res. 2014; 57(1):6-11. doi:10.1016/j.jspr.2014.01.001

13.Lorini I, Krzyzanowski FC, França-Neto JB, Henning AA. Principais pragas e métodos de controle em sementes durante o armazenamento - Série sementes. Londrina: EMBRAPA-CNPS; 2009.

14.Lee SE, Lee BH, Choi WS, Park BS, Kim JG, Campbell B. Fumigant toxicity of volatile natural products from Korean spicies and medicinal plants towards the rice weevil, Sitophilus oryzae (L.). Pest Manag Sci. 2001; 57(6):548-53. doi:10.1002/ps.322

15. Isman MB. Botanical insecticides, deterrents, and repellents in modern agriculture and an increasingly regulated world. Annu Rev Entomol. 2006; 51 (1):45-66. doi:10.1146/annurev.ento.51.110104.151146 
16.Isman MB. Botanical insecticides: for richer, for poorer. Pest Manag Sci. 2008; 64(1):8-11. doi:10.1002/ps.1470

17.Martinez S, Van Emden H. Growth disruption, abnormalities and mortality of Spodoptera littoralis (Boisduval) (Lepidoptera: Noctuidae) caused by azadirachtin. Neotrop Entomol. 2001; 30(1):113-24. doi:10.1590/S1519$566 \times 2001000100017$

18.Rattan RS. Mechanism of action of insecticidal secondary metabolites of plant origin. Crop Prot. 2010; 29(9):913-20. doi:10.1016/j.cropro.2010.05.008

19.Kim KH, Kabir E, Jahan SA. Exposure to pesticides and the associated human health effects. Sci Total Environ. 2017; 575(1):525-35. doi:10.1016/j. scitotenv.2016.09.009

20.Corrêa J, Salgado HRN. Atividade inseticida das plantas e aplicações: revisão. Rev. Bras. Plantas Med. 2011; 13(4):500-6. doi:10.1590/S1516-05722011000400016

21.Dayan FE, Cantrell CL, Duke SO. Natural products in crop protection. Bioorg Med Chem. 2009; 17(12):4022-34. doi:10.1016/j.bmc.2009.01.046

22.Gonçalves GLP, Ribeiro LP, Gimenes L, Vieira PC, Silva MFGF, Forim MR, Fernandes JB, Vendramim JB. Lethal and sublethal toxicities of Annona sylvatica (Magnoliales: Annonaceae) extracts to Zabrotes subfasciatus (Coleoptera: Chrysomelidae: Bruchinae). Fla Entomol. 2015; 98(3):921-8. doi:10.1653/024.098.0317

23.Regnault-Roger C, Vincent C, Arnason JT. Essential oils in insect control: lowrisk products in a high-stakes world. Annu Rev Entomol. 2012; 57(1):405-24. doi:10.1146/annurev-ento-120710-100554

24. Ribeiro LP, Vendramim JD, Bicalho KU, Andrade MS, Fernandes JB, Moral RA, 
Demétrio CGB. Annona mucosa Jacq. (Annonaceae): a promising source of bioactive compounds against Sitophilus zeamais Mots. (Coleoptera:

Curculionidae). J Stored Prod Res. 2013; 55(1):6-14. doi:10.1016/j.jspr.2013.06.001

25. Oliveira JV, Vendramim JD. Repelência de óleos essenciais e pós vegetais sobre adultos de Zabrotes subfasciatus (Boh.) (Coleoptera: Bruchidae) em sementes de feijoeiro. Ann. Entomol. Soc. Am. 1999; 28(3):549-55. doi:10.1590/ S0301-80591999000300026

26.Mazzonetto F, Vendramim J. Efeito de pós de origem vegetal sobre Acanthoscelides obtectus (Say) (Coleoptera: Bruchidae) em feijão armazenado. Neotrop Entomol. 2003; 32(1):145-9.

27.Almeida FAC, Goldfarb AC, Gouveia JPG. Avaliação de extratos vegetais e métodos de aplicação no controle de Sitophilus spp. Rev Bras Prod Agroind. 1999; 1 (1):13-20.

28. Santos-Rando JS, Lima CB, Batista NA, Feldhaus DC, Lourenço CC, Polonio VD, Ávila RR, Malanotte ML. Extratos vegetais no controle dos afídeos Brevicoryne brassicae (L.) e Myzus persicae (Sulzer). Semina: Ciênc. Agrár. 2011 ; 32(2):503-11. doi:10.5433/1679-0359.2011v32n2p503

29.Fabro JR, Rabelo AK, Grisa F. Métodos ecológicos de controle de insetos e de doenças das plantas e dos solos. Associação de Estudos, Orientação e Assistência Rural; Centro do Apoio ao Pequeno Agricultor. Francisco Beltrão: ASSESOAR; 2014.

30.Ferreira DF. SISVAR: um programa para análises e ensino de estatística. Rev Symposium 2008; 6(2):36-41. 
31.Lin H, Kogan M, Fischer D. Induced resistance in soybean to the Mexican bean beetle (Coleoptera: Coccinellidae): comparisons of inducing factors. Environ Entomol. 1990; 19(6):1852-7. doi:10.1093/ee/19.6.1852

32.Scott IM, Jensen HR, Philogène BJ, Arnason JT. A review of Piper spp. (Piperaceae) phytochemistry, insecticidal activity and mode of action. Phytochem Rev. 2008; 7(1):65. doi:10.1007/s11101-006-9058-5

33. Awoyinka OA, Oyewole IO, Amos BMW, Onasoga OF. Comparative pesticidal activity of dichloromethane extracts of Piper nigrum against Sitophilus zeamais and Callosobruchus maculatus. Afr. J. Biotechnol. 2006; 5(24):2446-9.

34.Procópio SO, Vendramim JD, Ribeiro-Júnior JI, Santos JB. Efeitos de pós vegetais sobre Acanthoscelides obtectus (Say) e Zabrotes subfasciatus (Boh.) (Coleoptera: Bruchidae). Ver Ceres 2003; 50(289):395-405.

35. Salvadores YU, Silva GA, Tapia MV, Hepp RG. Spices powders for the control of maize weevil, Sitophilus zeamais Motschulsky, in stored wheat. Agric Técnica 2007; 67(2):147-54.

36.Mishra BB, Tripathi SP. Repellent activity of plant derived essential oils against Sitophilous oryzae (Linnaeus) and Tribolium castenium (Herbst). Singapore J Scientific Res 2011 ; 1 (2):173-8. doi:10.3923/sjsres.2011.173.178

37. Akhtar $Y$, Isman MB. Plant natural products for pest management: the magic of mixtures. In Ishaaya I, Palli SR, Horowitz AR, editores. Advanced technologies for managing insect pests. Dordrecht: Springer; 2013. p. 231-247.

38.Cantrell CL, Dayan FE, Duke SO. Natural products as sources for new pesticides. J Nat Prod. 2012; 75(6):1231-42. doi:10.1021/np300024u

39.Ribeiro LP et al. Pimenta pseudocaryophyllus derivatives: extraction methods and bioactivity against Sitophilus zeamais Motschulsky (Coleoptera: 
Curculionidae). Neot Entomol. 2015; 44(6):634-42. doi:10.1007/s13744-015-0321-6 Data de submissão: 10 de abril de 2018 Avaliado em: 20 de abril de 2018 (AVALIADOR A) Avaliado em: 12 de abril de 2018 (AVALIADOR B) Aceito em: 25 de abril de 2018 